\title{
Non-equilibrium beta processes in superfluid neutron star cores
}

\author{
L. Villain ${ }^{1,2}$ and P. Haensel ${ }^{1}$ \\ 1 N. Copernicus Astronomical Center, Polish Academy of Sciences, Bartycka 18, 00-716 Warszawa, Poland \\ e-mail: loic@camk.edu.pl \\ 2 LUTH, UMR 8102 du CNRS, Observatoire de Paris-Meudon, 92195 Meudon Cedex, France
}

Received 26 April 2005 / Accepted 28 July 2005

\begin{abstract}
The influence of nucleons superfluidity on the beta relaxation time of degenerate neutron star cores, composed of neutrons, protons and electrons, is investigated. We numerically calculate the implied reduction factors for both direct and modified Urca reactions, with isotropic pairing of protons or anisotropic pairing of neutrons. We find that due to the non-zero value of the temperature and/or to the vanishing of anisotropic gaps in some directions of the phase-space, superfluidity does not always completely inhibit beta relaxation, allowing for some reactions if the superfluid gap amplitude is not too large in respect to both the typical thermal energy and the chemical potential mismatch. We even observe that if the ratio between the critical temperature and the actual temperature is very small, a suprathermal regime is reached for which superfluidity is almost irrelevant. On the contrary, if the gap is large enough, the composition of the nuclear matter can stay frozen for very long durations, unless the departure from beta equilibrium is at least as important as the gap amplitude. These results are crucial for precise estimation of the superfluidity effect on the cooling/slowing-down of pulsars and we provide online subroutines to be implemented in codes for simulating such evolutions.
\end{abstract}

Key words. dense matter - equation of state - stars: neutron

\section{Introduction}

In the simplest model, the so-called npe matter model, neutron star (NS) cores are transparent for neutrinos and mainly consist of degenerate neutrons, with a small admixture of equal numbers of protons and electrons. In such conditions, the composition is characterized by the fraction of protons among the total number of nucleons, $x_{p}$. Due to beta reactions, the value of $x_{p}$ depends on density and is usually determined by the condition of beta equilibrium, for instance in the calculations of non-dynamical quantities, such as the equation of state (EOS). However, the assumption of beta equilibrium is only valid if the density of a matter element changes on a timescale $\tau_{\rho}$ much longer than the beta equilibration timescale $\tau_{\beta}$. But in strongly degenerate npe matter, $\tau_{\beta}$ is made much longer than in normal matter by the decrease of the available phase-space due to the Pauli exclusion principle. As a consequence, various astrophysical scenarios in which the condition $\tau_{\beta} \ll \tau_{\rho}$ is violated were pointed out by several authors. Such a situation occurs in gravitational collapse of neutron star (Haensel 1992; Gourgoulhon \& Haensel 1993), during pulsar spin-down (Reisenegger 1995, 1997; Fernández \& Reisenegger 2005), or in the neutron star interiors just due to the existence of even relatively slow hydrodynamic flows (for an example, see Urpin \& Shalybkov 1996) or of millisecond oscillations (Reisenegger \& Goldreich 1992; Villain et al. 2005).
Nonetheless, beta equilibrium breaking and change of composition are crucial to take into account not only from those mainly "mechanical" points of view, but also in the thermal evolution of not too old neither too young NSs. Indeed, the cooling of such NSs proceeds through the emission of neutrinos by the cold (i.e., degenerate) npe matter, whose deviation from beta equilibrium is characterized by the chemical potential mismatch $\delta \mu \equiv \mu_{n}-\mu_{p}-\mu_{p}$. While in beta equilibrium only the non-zero value of the temperature $T$ determines the size of the available phase-space, and consequently all reactions rates, $\delta \mu \neq 0$ opens additional volume in the phase-space for beta processes. This results in an increase of the neutrino emissivity, and as beta reactions occurring off-equilibrium produce entropy and therefore heat neutron star matter, the net effect is the matter heating (Haensel 1992; Gourgoulhon \& Haensel 1993; Reisenegger 1995, 1997; Fernández \& Reisenegger 2005).

Furthermore, the description of any basic physical process that takes place inside NSs can be made more difficult by another phenomenon, which is the possible superfluidity of nucleons. Indeed, it is expected that in about one year after neutron star birth, neutrons (protons) in the core become superfluid, when temperature goes below critical one, $T_{\mathrm{c} n}\left(T_{\mathrm{c} p}\right)$. Then, energy gaps, $\Delta_{n}$ and $\Delta_{p}$, appear in the nucleon excitation energy spectra, reducing again the available phase-space. This strongly slows down the beta processes in dense matter for $T \ll T_{\mathrm{c}}$ and 
also makes the dynamical properties, such as pulsations (Lee 1995; Andersson \& Comer 2001; Prix \& Rieutord 2002), more complicated to evaluate.

To summarize, the beta processes rate, in its full complexity, involves at least four parameters with the dimension of energy: $\delta \mu, k_{\mathrm{B}} T, \Delta_{n}$, and $\Delta_{p}$. The roles of the first and the second pairs of parameters are opposite: $\delta \mu, k_{\mathrm{B}} T$ increase the available phase-space, while the superfluid energy gaps inhibit the reactions. In the limit of small deviations from the thermodynamic equilibrium, $\delta \mu / k_{\mathrm{B}} T \ll 1$, the beta relaxation rate can be linearized in this small parameter and the dissipation can be represented by the bulk viscosity (Haensel et al. 2000, 2001, 2002, and references therein). Nevertheless, in the general case, both the heating rate and neutrino losses have to be calculated explicitly in the out of beta equilibrium npe matter. For a non-superfluid matter this was done in Haensel (1992), Gourgoulhon \& Haensel (1993), Reisenegger (1995) and Fernández \& Reisenegger (2005). The effect of nucleon superfluidity on beta relaxation rates was studied using a very crude model by Reisenegger (1997). He considered deviation from beta equilibrium implied by the the pulsar spin-down, and assumed that for $\delta \mu<\Delta_{p}+\Delta_{n}$ the beta reactions were completely blocked (no reactions), while for $\delta \mu>\Delta_{p}+\Delta_{n}$ the effect of superfluidity could be neglected (normal matter rates). As we should discuss in more details later, such a step-like modeling of the reduction factor behaviour is unrealistic.

In the present article, we calculate numerically the superfluid reduction factors for a broad range of the relevant parameters making also available on-line subroutines to proceed to any farther calculation. Both direct Urca and modified Urca processes are considered in the presence of various types of superfluidity. In this way, we get correct reduction factors that can be used for simulating the evolution of superfluid neutron star cores which are off beta equilibrium.

The formulae for the rates of non-equilibrium beta processes in a non-superfluid npe matter are reminded in Sect. 2. Basic features of nucleon superfluidity in NSs are presented in Sect. 3. In Sect. 4, the formulae for the superfluid reduction factors are derived, with some illustrative numerical results in Sect. 5. In Sect. 6, we discuss our results and their possible application in numerical simulations of neutron star evolution and dynamics. Finally, in the Appendix, the subroutines to calculate the reduction factors for such numerical simulations are briefly described, together with some comments on their practical use and the address of the website from which they can be downloaded.

\section{Out-of-equilibrium beta processes without superfluidity}

Non-equilibrium beta processes in normal (non superfluid) neutron star cores have already been the subject of several studies (Haensel 1992; Reisenegger 1995). In this section, we shall not discuss the astrophysical conditions in which the breaking of chemical equilibrium can occur, but focus on its microphysical description. Moreover, we just give here a brief summary of known results, introducing our notations in units
$\hbar=k_{\mathrm{B}}=c=1$ (with exception of Sect. 3), mainly following the conventions of Haensel (1992).

We take the basic npe model of nuclear NS matter and assume that

- the core's content is in a stationary state (no oscillation), with all particles comoving. The outcome of a possible difference between the motions (e.g., rotation) of $n$ and $p$ fluids (a crucial phenomenon for superfluids) will be considered elsewhere;

- the nucleons are strongly interacting non-relativistic Fermi liquids and the electrons form an ultrarelativistic ideal Fermi gas. The temperature is sufficiently low for the npe components to be strongly degenerate, and we shall then work for all of them with quantities at zero temperature and at thermodynamic, but not necessarily chemical, equilibrium: beta equilibrium is not assumed. Hence, the Fermi momenta $p_{\mathrm{F} i}$, for the $i$ species, versus the respective densities $n_{i}$, are $p_{\mathrm{F} i}=\left(3 \pi^{2} n_{i}\right)^{1 / 3}$;

- local electromagnetic equilibrium is reached and matter is electrically neutral;

- the neutrinos freely escape from the star.

Cooling of NSs involves various reactions, among which the Urca processes are the only ones that we should deal with in this article. Indeed, as neutrinos freely escape from the star, other reactions, such as bremsstrahlung, do not change the chemical composition of npe matter and are then not affected by beta equilibrium breaking. Yet, a distinction has to be made between two types of Urca processes, since the direct Urca reactions (called thereafter Durca, following K. Levenfish, see, Yakovlev et al. 2001) are kinematically allowed only if the proton fraction is high enough. If not, the modified Urca reactions (Murca) ${ }^{1}$ play the key-role, nonetheless they can be neglected when Durca occur. From a practical point of view, it means that there is a threshold density below which one has to deal with (and only with) Murca reactions, while, for densities above that value, it is sufficient to consider only Durca processes. As the latter are the simplest, we shall start with their description.

\subsection{Direct Urca processes}

In $n p e$ matter, Durca reactions are allowed if $p_{\mathrm{F} n}<p_{\mathrm{F} p}+p_{\mathrm{Fe}}$. Assuming local neutrality of matter, it is equivalent to $p_{\mathrm{F} n}<2 p_{\mathrm{F} p}$ and then to $n_{p}>n / 9$, where $n$ is the total baryonic density $n=n_{p}+n_{n}$. This leads to a threshold value of the density that depends on the equation of state, and mainly on the symmetry energy, but which is typically several times the standard nuclear matter density $n_{0}=2.8 \times 10^{14} \mathrm{~g} \mathrm{~cm}^{-3}$.

When beta equilibrium is assumed, the two Durca reactions,

$D_{n}: \quad n \rightarrow p+e^{-}+\bar{v}_{e}$,

$D_{p}: \quad p+e^{-} \rightarrow n+v_{e}$,

have the same rates and form, all together, the usual Durca process. As we do not assume beta equilibrium, we have to

\footnotetext{
1 With, in respect to Durca, an additional spectator nucleon.
} 
consider two different rates (numbers of reactions per $\mathrm{cm}^{3}$ and during one second) (see Haensel 1992; Reisenegger 1995)

$$
\begin{aligned}
\Gamma_{D_{n}}= & \int \frac{\mathrm{d} \boldsymbol{p}_{e}}{(2 \pi)^{3}} \frac{\mathrm{d} \boldsymbol{p}_{p}}{(2 \pi)^{3}} \frac{\mathrm{d} \boldsymbol{p}_{n}}{(2 \pi)^{3}} \frac{\mathrm{d} \boldsymbol{p}_{v}}{(2 \pi)^{3}}\left(1-f_{e}\right)\left(1-f_{p}\right) f_{n} \\
& \times(2 \pi)^{4} \delta\left(E_{f}-E_{i}\right) \delta\left(\boldsymbol{P}_{f}-\boldsymbol{P}_{i}\right)\left|M_{i f}\right|^{2}
\end{aligned}
$$

and

$$
\begin{aligned}
\Gamma_{D_{p}}= & \int \frac{\mathrm{d} \boldsymbol{p}_{e}}{(2 \pi)^{3}} \frac{\mathrm{d} \boldsymbol{p}_{p}}{(2 \pi)^{3}} \frac{\mathrm{d} \boldsymbol{p}_{n}}{(2 \pi)^{3}} \frac{\mathrm{d} \boldsymbol{p}_{v}}{(2 \pi)^{3}}\left(1-f_{n}\right) f_{p} f_{e} \\
& \times(2 \pi)^{4} \delta\left(E_{f}-E_{i}\right) \delta\left(\boldsymbol{P}_{f}-\boldsymbol{P}_{i}\right)\left|M_{i f}\right|^{2} .
\end{aligned}
$$

In these equations, $\left|M_{i f}\right|^{2}$ is the squared transition amplitude summed and averaged on spins states, $f_{i} \widehat{\equiv}\left(1+\mathrm{e}^{\left(\varepsilon_{i}-\mu_{i}\right) / T}\right)^{-1}$ is the Fermi-Dirac distribution for a fermion $i$ with energy $\varepsilon_{i}$, chemical potential $\mu_{i}$, and temperature $T$, while the $\delta$-functions ensure the conservation of the total energy and momentum. The physical quantity we will focus on is $\Delta \Gamma_{D}$, that we define as the difference between $\Gamma_{D_{n}}$ and $\Gamma_{D_{p}}: \Delta \Gamma_{D} \widehat{=} \Gamma_{D_{n}}-\Gamma_{D_{p}}$. This quantity characterizes the relaxation rate (and hence timescale) for reaching beta equilibrium.

In the context of strongly degenerate matter, there is a standard approximation, that can be applied here, to go beyond the previous formulae in an analytical way, the so-called phase-space decomposition (see Shapiro \& Teukolsky 1983). It mainly consists in replacing in the integrals all smooth functions by their values at $p_{i}=p_{\mathrm{F} i}$, which enables to factorise out from the integrals the mean value of the microphysical factor $\left|M_{i f}\right|^{2}$. We shall not make explicit the whole calculation, and send the reader to Yakovlev et al. (2001) for a detailed review. However, with the following dimensionless variables $(i=n, p, e)$

$x_{i}=\frac{\varepsilon_{i}-\mu_{i}}{T}, \quad x_{v}=\frac{\varepsilon_{v}}{T}, \quad \xi=\frac{\delta \mu}{T}$,

where we defined $\delta \mu \widehat{\equiv} \mu_{n}-\mu_{p}-\mu_{e}$, we finally get

$\Delta \Gamma_{D}(\xi)=\Delta \Gamma_{D_{0}} I_{D}(\xi)$,

with the dimensionless integral

$$
\begin{aligned}
I_{D}(\xi)= & \int_{0}^{\infty} \mathrm{d} x_{v} x_{v}^{2} \int_{-\infty}^{+\infty} \mathrm{d} x_{n} \mathrm{~d} x_{p} \mathrm{~d} x_{e} \\
& \left\{f_{n}\left(1-f_{p}\right)\left(1-f_{e}\right) \delta\left(x_{n}-x_{p}-x_{e}-x_{v}+\xi\right)\right. \\
& \left.-f_{p} f_{e}\left(1-f_{n}\right) \delta\left(x_{n}+x_{v}-x_{p}-x_{e}+\xi\right)\right\} .
\end{aligned}
$$

The rate $\Delta \Gamma_{D_{0}}$, a physical factor that depends on the EOS, takes such typical values that the relaxation time, once the integral (6) calculated, is around $\tau_{\text {rel }}^{(D)} \sim 20 T_{9}^{-4} \mathrm{~s}$, with $T_{9}=$ $T /\left(10^{9} \mathrm{~K}\right)$ (Yakovlev et al. 2001).

As far as the calculation of the integral (6) is concerned, using $f\left(x_{i}\right) \widehat{=} f_{i}=1-f\left(-x_{i}\right)$ combined with known results on Fermi integrals, we are lead to

$I_{D}(\xi)=\int_{0}^{\infty} \mathrm{d} x_{v} x_{v}^{2}\left\{J_{\mathrm{D}}\left(x_{v}-\xi\right)-J_{\mathrm{D}}\left(x_{v}+\xi\right)\right\}$, where $J_{\mathrm{D}}(x) \widehat{\equiv} f(x)\left(x^{2}+\pi^{2}\right) / 2$. The latter integration can now be done analytically and gives (Reisenegger 1995)

$$
I_{D}(\xi)=\xi \pi^{4} \frac{17}{60}\left(1+\frac{10 \xi^{2}}{17 \pi^{2}}+\frac{\xi^{4}}{17 \pi^{4}}\right) .
$$

\subsection{Modified Urca}

Durca processes are by many orders of magnitude the fastest beta processes in NS cores. However, as already mentioned, they can be kinematically forbidden. If this is the case, the Murca reactions prevail, which involve an additional spectator nucleon to ensure the conservation of both energy and momentum. This additional nucleon can be either a proton or a neutron. Hence, the distinction has to be made between two branches, and not only when some nucleons are superfluid. The Murca reactions are

$$
\begin{array}{ll}
M_{n}^{N}: & n+N \rightarrow p+N+e^{-}+\bar{v}_{e} \\
M_{p}^{N}: & p+N+e^{-} \rightarrow n+N+v_{e},
\end{array}
$$

where $N$ is the spectator nucleon. If this particle is a proton, these reactions are called proton branch of Murca, and they are called neutron branch whether it is a neutron ${ }^{2}$.

If none of the nucleons is superfluid, we do not really have to make distinction between the two branches in the phasespace integral, and using the same notations as in the Durca case (skipping the details of the calculations that are very similar), we can write

$$
\Delta \Gamma_{M}(\xi)=\Delta \Gamma_{M_{0}} I_{M}(\xi)
$$

with

$$
\begin{aligned}
I_{M}(\xi)= & \int_{0}^{\infty} \mathrm{d} x_{v} x_{v}^{2} \int_{-\infty}^{+\infty} \mathrm{d} x_{n} \mathrm{~d} x_{p} \mathrm{~d} x_{e} \mathrm{~d} x_{N \mathrm{i}} \mathrm{d} x_{N \mathrm{f}} \\
& \left\{f_{n} f_{N \mathrm{i}}\left(1-f_{p}\right)\left(1-f_{e}\right)\left(1-f_{N \mathrm{f}}\right)\right. \\
& \times \delta\left(x_{n}+x_{N \mathrm{i}}-x_{p}-x_{e}-x_{N \mathrm{f}}-x_{v}+\xi\right) \\
& -f_{p} f_{e} f_{N \mathrm{i}}\left(1-f_{n}\right)\left(1-f_{N \mathrm{f}}\right) \\
& \left.\times \delta\left(x_{n}+x_{N \mathrm{f}}+x_{v}-x_{p}-x_{N \mathrm{i}}-x_{e}+\xi\right)\right\} .
\end{aligned}
$$

Here, $x_{N \mathrm{i}}$ and $x_{N \mathrm{f}}$ are respectively the " $x$ variables", defined as in Eq. (4), for the initial and final spectator nucleons. Finally, $\Delta \Gamma_{M_{0}}$ is a constant that depends on the EOS and takes such values that the relaxation timescale is $\tau_{\text {rel }}^{(M)} \sim T_{9}^{-6}$ months.

Equation (11) can also be written

$I_{M}(\xi)=\int_{0}^{\infty} \mathrm{d} x_{v} x_{v}^{2}\left\{J_{\mathrm{M}}\left(x_{v}-\xi\right)-J_{\mathrm{M}}\left(x_{v}+\xi\right)\right\}$

where $J_{\mathrm{M}}(x) \widehat{\equiv} f(x)\left(x^{4}+10 \pi^{2} x^{2}+9 \pi^{4}\right) / 24$, which leads to (Reisenegger 1995)

$I_{M}(\xi)=\frac{367 \xi \pi^{6}}{1512}\left(1+\frac{189 \xi^{2}}{367 \pi^{2}}+\frac{21 \xi^{4}}{367 \pi^{4}}+\frac{3 \xi^{6}}{1835 \pi^{6}}\right)$

\footnotetext{
${ }^{2}$ Notice that the proton branch has a density threshold, but it is much smaller than the threshold for Durca, with the condition $n_{p}>$ $n / 65$ in npe matter (Yakovlev \& Levenfish 1995).
} 
In the presence of superfluidity, the rates can be much more complicated to calculate. Indeed, in all the previous calculations, we could quite easily get rid of all angular integrals as they always gave contributions that could be factorised out. The factorisation was made possible since the only function that depends on the respective directions of momenta was, a priori, the square of the transition amplitudes. Indeed, it can be shown, either in the Durca or in the Murca case, that it is a proper approximation to use some suitably defined angle averaged values (Friman \& Maxwell 1979; Yakovlev \& Levenfish 1995) of $\left|M_{i f}\right|^{2}$, instead of doing the full angular integral. However, this approximation can be no longer sufficient to factorise the angular integrals if some nucleons are superfluid. This results from the fact that the Cooper pairing can occur in anisotropic states, a feature that is explained in the next section dealing with nucleon superfluidity in NS cores.

\section{Superfluidity of nucleons}

Superfluidity of nucleons in NS cores is reviewed by Lombardo $\&$ Schulze (2001) and we shall only give here a brief summary of the results useful in the following. Neutrons are believed to form Cooper pairs due to their interaction in the triplet ${ }^{3} \mathrm{P}_{2}$ state, while protons form singlet ${ }^{1} \mathrm{~S}_{0}$ pairs. In the study of the triplet-state neutron pairing, one should distinguish the cases of the different possible projections $m_{J}$ of $n n$-pair angular momentum $\boldsymbol{J}$ onto a quantization axis $z$ (see, e.g., Amundsen \& Østgaard 1985): $\left|m_{J}\right|=0,1,2$. The actual (energetically most favorable) state of $n n$-pairs is not known, being extremely sensitive to the (still unknown) details of $n n$ interaction. One cannot exclude that this state varies with density and is a superposition of states with different $m_{J}$.

Hence, in the following, we shall conform ourselves to what is usually done in the community (see, e.g., Yakovlev et al. 2001) and deal with three different superfluidity types: ${ }^{1} \mathrm{~S}_{0},{ }^{3} \mathrm{P}_{2}\left(m_{J}=0\right)$ and ${ }^{3} \mathrm{P}_{2}\left(\left|m_{J}\right|=2\right)$, denoted as $\mathrm{A}, \mathrm{B}$ and $\mathrm{C}$, respectively (Table 1 ). The superfluidity of type $\mathrm{A}$ is attributed to protons, while types $\mathrm{B}$ and $\mathrm{C}$ may be attributed to neutrons and are sufficient to have a general idea about possible impact of neutrons superfluidity since in these two cases its effect are qualitatively different.

In addition, it can be shown (see, e.g., Yakovlev et al. 2001) that in our context, it is sufficient, from the microscopic point of view, to consider as the only effect of superfluidity the introduction of an energy gap $\delta$ in momentum dependence of the nucleon energy, $\varepsilon(\boldsymbol{p})$. Near the Fermi level $\left(\left|p-p_{\mathrm{F}}\right| \ll p_{\mathrm{F}}\right)$, this dependence can be written as

$$
\begin{aligned}
& \varepsilon=\mu-\sqrt{\delta^{2}+v_{\mathrm{F}}^{2}\left(p-p_{\mathrm{F}}\right)^{2}} \text { at } p<p_{\mathrm{F}}, \\
& \varepsilon=\mu+\sqrt{\delta^{2}+v_{\mathrm{F}}^{2}\left(p-p_{\mathrm{F}}\right)^{2}} \text { at } p \geq p_{\mathrm{F}},
\end{aligned}
$$

where $p_{\mathrm{F}}$ and $v_{\mathrm{F}}$ are the Fermi momentum and Fermi velocity of the nucleon, respectively, while $\mu$ is the nucleon chemical potential. One has $\delta^{2}=\Delta^{2}(T) F(\vartheta)$, where $\Delta(T)$ is the part of the gap's amplitude that depends on the temperature, and $F(\vartheta)$ specifies dependence of the gap on the angle $\vartheta$ between the particle momentum and the $z$ axis (Table 1). In case A the gap
Table 1. Three types of superfluidity. From Haensel et al. (2000) with the kind permission of the authors.

\begin{tabular}{c|ccc}
\hline \hline Type & Pairing state & $F(\vartheta)$ & $k_{\mathrm{B}} T_{\mathrm{c}} / \Delta(0)$ \\
\hline $\mathrm{A}$ & ${ }^{1} \mathrm{~S}_{0}$ & 1 & 0.5669 \\
$\mathrm{~B}$ & ${ }^{3} \mathrm{P}_{2}\left(m_{J}=0\right)$ & $\left(1+3 \cos ^{2} \vartheta\right)$ & 0.8416 \\
$\mathrm{C}$ & ${ }^{3} \mathrm{P}_{2}\left(\left|m_{J}\right|=2\right)$ & $\sin ^{2} \vartheta$ & 0.4926 \\
\hline
\end{tabular}

is isotropic, and $\delta=\Delta(T)$. In cases B and C, the gap depends on $\vartheta$. Note that in case $\mathrm{C}$ the gap vanishes at the poles of the Fermi sphere at any temperature: $F_{\mathrm{C}}(0)=F_{\mathrm{C}}(\pi)=0$.

Notice also that the gap amplitude, $\Delta(T)$, is derived from the standard equation of the BCS theory (see, e.g., Yakovlev et al. 1999), with the value of $\Delta(0)$ that determines the critical temperature $T_{\mathrm{c}}$. The values of $k_{\mathrm{B}} T_{\mathrm{c}} / \Delta(0)$ for cases $\mathrm{A}, \mathrm{B}$ and $\mathrm{C}$ are given in Table 1.

For further analysis it is convenient to introduce the dimensionless quantities:

$v=\frac{\Delta(T)}{k_{\mathrm{B}} T}, \quad \tau=\frac{T}{T_{\mathrm{c}}}, \quad y=\frac{\delta}{k_{\mathrm{B}} T}$.

The dimensionless gap $y$ can be presented in the form:

$y_{\mathrm{A}}=v_{\mathrm{A}}, \quad y_{\mathrm{B}}=v_{\mathrm{B}} \sqrt{1+3 \cos ^{2} \vartheta}, \quad y_{\mathrm{C}}=v_{\mathrm{C}} \sin \vartheta$

where the dimensionless gap amplitude $v$ depends only on $\tau$. In case A the quantity $v$ coincides with the isotropic dimensionless gap, while in cases B and C it represents, respectively, the minimum and maximum gap (as a function of $\vartheta$ ) on the nucleon Fermi surface. As we shall see later, this implies that for global integrated quantities (such as rates), with a given value of $v$, one can expect a stronger effect of superfluidity for the case B and a weaker for the case $\mathrm{C}$, while the case A should be in between. Notice finally that the dependence of $v$ on $\tau$ was fitted by Levenfish \& Yakovlev (1994). However, their fits will not be used in the following. Indeed, we shall only deal with microscopic calculations and not with astrophysical applications, and we shall therefore use $v$ as a free parameter.

\section{Out of equilibrium beta processes with superfluidity}

\subsection{General features of the effect of superfluidity on reactions rates}

The influence of superfluidity on the beta relaxation time was already investigated by Reisenegger (1997), but only in a very rough way. The principle that he used is based on what was explained in Sect. 3, i.e., on the fact that to estimate this influence on averaged physical quantities, such as emissivity or reaction rates, it is sufficient, at the first level of approximation, to only consider the creation, near the Fermi surface, of a gap in the dispersion relation $\varepsilon_{i}=\varepsilon_{i}(p)$. This property can be demonstrated using Bogoliubov transformations (see, e.g., Yakovlev et al. 2001) and has, as a first obvious consequence, strong decrease of the available phase-space. Hence, as Reisenegger 
concluded, this can result in a blocking of the reactions during quite long times, even if chemical equilibrium is broken. Nevertheless, there are two shortcomings in the assumption of a completely frozen composition, as made by Reisenegger. First, the temperature is not exactly null, which implies the existence of excited states and, therefore, of some reactions. Second, the Cooper pairing does not always occur in a singlet spin state, which has for consequence a possible anisotropy of the gap and of the Fermi surface, allowing for reactions in some directions of the momentum space.

Thus, to improve the study of Reisenegger (1997), we shall apply in the following the procedure that consists in adding a gap close to the Fermi surface in the dispersion relation, but do the calculations of relaxation rates through integrals similar to those described in Sect. 2. In practice, it means, for superfluid nucleons, to replace in the integrals the usual dimensionless variables $x_{i}$ by $z_{i}$, with

$z_{i} \widehat{=} \frac{\varepsilon_{i}-\mu_{i}}{T}=\operatorname{sign}\left(x_{i}\right) \sqrt{x_{i}^{2}+y_{i}^{2}}$,

where

$x_{i} \widehat{=} \frac{v_{\mathrm{F} i}\left(p_{i}-p_{\mathrm{F} i}\right)}{T} \quad$ and $\quad y_{i} \widehat{=} \frac{\delta_{i}(T, \vartheta)}{T} \widehat{=} v_{i}(T) \mathrm{F}_{\mathrm{i}}(\vartheta)$.

In these expressions,

$-v_{\mathrm{F} i}=\left(\partial \varepsilon_{i} / \partial p_{i}\right)_{p_{i}=p_{\mathrm{F} i}}$ is the Fermi velocity, making the definitions for $x_{i}$, Eqs. (4) and (18), equivalent near the Fermi surface when there is no gap;

- $\delta_{i}(T, \vartheta)$ is the superfluid gap with $T$ the temperature and $\vartheta$ the angle between the particle momentum and the quantization axis (see Sect. 3);

$-v_{i}(T)$ is the dimensionless gap amplitude function and $\mathrm{F}_{\mathrm{i}}(\vartheta)$ the dimensionless anisotropy function with, for the isotropic case $(\mathrm{A}), \mathrm{F}_{\mathrm{i}}(\vartheta) \equiv 1$ (see Sect. 3).

The next issue that needs to be clarified before doing any calculation is the identification of the relevant cases to deal with. For instance, as it is well-known that protons can only pair in spin-singlets, there is no need to evaluate the effect of two simultaneous anisotropic pairings. Furthermore, a first approximation, which should not be too bad, is to assess that there is always only one type of superfluid nucleons, which is equivalent to assume that the dominant gap prevails. Hence, we shall in the following only study cases with superfluidity of one type of nucleons, beginning with Durca processes that involves less nucleons than Murca processes. Finally, notice that even if our goal is to calculate the superfluid equivalent of the rates (5) and (10), we are able, with the phase-space approximation, to extract from these rates all microphysical uncertainties $\left(\Delta \Gamma_{D_{0}}\right.$ and $\Delta \Gamma_{M_{0}}$ ), leaving dimensionless integrals $\left[I_{D}(\xi)\right.$ and $\left.I_{M}(\xi)\right]$. As a consequence, in order to also minimize, in the superfluid cases, the uncertainties coming from microphysics, it is more relevant not to directly calculate the superfluid rates, but the ratios between their values with and without superfluidity. In such a way, only uncertainties linked with the gap still affect the phase-space integrals and the results.

This leads us to write, for all type of reactions, equations like

$\Delta \Gamma_{X}^{i}=\Delta \Gamma_{X_{0}} I_{X}^{i}\left(\xi, v_{j}\right)=\Delta \Gamma_{X} R_{X}^{i}\left(\xi, v_{j}\right)$, where $R_{X}^{i}\left(\xi, v_{j}\right) \widehat{\equiv} I_{X}^{i}\left(\xi, v_{j}\right) / I_{X}(\xi)$ are the quantities we shall estimate: the reduction factors for the $X$ type of reactions. In this expression, $i$ labels the type of superfluidity (A, B or C), and $j$ the type of nucleon that is superfluid ( $n$ or $p$ ) with a gap $v_{j}$ (see definition in Eq. (18)). Finally, notice that by definition, for $T$ greater than both critical temperatures, one has $R_{X}^{i}\left(\xi, v_{j}\right)=R_{X}^{i}(\xi, 0)=1$, and for $T$ smaller than at least one critical temperature $R_{X}^{i}\left(\xi, v_{j}\right)<1$.

\subsection{Durca}

Taking into account the fact that in the Durca case with only one type of superfluidity there might be only one type of anisotropy induced by pairing, we can write the reduction factor $R_{D}^{i}\left(\xi, v_{j}\right)$ as

$$
\begin{aligned}
R_{D}^{i}\left(\xi, v_{j}\right) & =\frac{1}{I_{D}(\xi)} \int^{\pi / 2} \frac{\mathrm{d} \Omega}{4 \pi} H_{D}^{i}\left(\xi, v_{j}\right) \\
& =\frac{1}{I_{D}(\xi)} \int_{0}^{\pi} \mathrm{d} \vartheta \sin (\vartheta) H_{D}^{i}\left(\xi, v_{j}\right),
\end{aligned}
$$

where, for type $A$ of superfluidity, $H_{D}^{i}\left(\xi, v_{j}\right)$ does not depend on the $\vartheta$ angle between the momentum of the Cooper pair and the quantization axis.

Following Eq. (6), one then has

$$
\begin{aligned}
H_{D}^{i}\left(\xi, v_{j}\right) \widehat{\equiv} & \int_{0}^{\infty} \mathrm{d} x_{v} x_{v}^{2} \int_{-\infty}^{+\infty} \mathrm{d} x_{n} \mathrm{~d} x_{p} \mathrm{~d} x_{e} \\
& \times\left\{f_{n}\left(1-f_{p}\right)\left(1-f_{e}\right) \delta\left(z_{n}-z_{p}-x_{e}-x_{v}+\xi\right)\right. \\
& \left.-f_{p} f_{e}\left(1-f_{n}\right) \delta\left(z_{n}+x_{v}-z_{p}-x_{e}+\xi\right)\right\},
\end{aligned}
$$

where the $z$ variable for the non-superfluid nucleon has to be replaced with the corresponding $x$ variable, while $v_{j}$ is "included" in the $z$ variable for the superfluid nucleon and $f_{p}=f\left(z_{p}\right)=\left(1+\mathrm{e}^{z_{p}}\right)^{-1}$ with a similar definition for $f_{n}$.

The integration over the electron variable can easily be done due to the Dirac functions, and then, using the well-known formula for Fermi integrals,

$\int_{-\infty}^{+\infty} \mathrm{d} x f(x) f(y-x)=\frac{y}{\mathrm{e}^{y}-1} \widehat{=} G(y)$,

enables us to integrate over the $x$ variable for the non-superfluid nucleon, giving

$$
\begin{aligned}
H_{D}^{i}\left(\xi, v_{j}\right)= & \int_{0}^{\infty} \mathrm{d} x_{v} x_{v}^{2} \int_{0}^{+\infty} \mathrm{d} x_{j} \\
& \times\left\{f\left(z_{j}\right) G\left(x_{v}-\xi-z_{j}\right)+f\left(-z_{j}\right) G\left(x_{v}-\xi+z_{j}\right)\right. \\
& \left.-f\left(z_{j}\right) G\left(x_{v}+\xi-z_{j}\right)-f\left(-z_{j}\right) G\left(x_{v}+\xi+z_{j}\right)\right\}
\end{aligned}
$$

This formula is the simplest that can be reached analytically and will be evaluated with numerical methods (see Sect. 5). 


\subsection{Murca}

Whatever the situation with superfluidity, the main change between Durca and Murca reactions is the presence of spectator nucleons that make the calculations more difficult. Nevertheless, a great difference is that, in the superfluid case, the additional dimensions of the integral no longer lead to calculations that can quite easily be done analytically, mainly due to anisotropies.

Anyway, with the same method as previously described, Eq. (10) is now replaced with

$\Delta \Gamma_{M_{X}}^{i}=\Delta \Gamma_{M_{X 0}} I_{M_{X}}^{i}\left(\xi, v_{j}\right)=\Delta \Gamma_{M_{X}} R_{M_{X}}^{i}\left(\xi, v_{j}\right)$,

with $R_{M_{X}}^{i}\left(\xi, v_{j}\right)=I_{M_{X}}^{i}\left(\xi, v_{j}\right) / I_{M}(\xi)$, while $X$ is the index of the branch ( $n$ or $p$ ), $i$ the index of the superfluidity type and $j$ the index of the superfluid nucleon. Here again, for $T$ greater than the critical temperature, one has $R_{M_{X}}^{i}\left(\xi, v_{j}\right)=R_{M_{X}}^{i}(\xi, 0)=1$ and in other cases $R_{M_{X}}^{i}\left(\xi, v_{j}\right)<1$.

Notice that $I_{M_{X}}^{i}\left(\xi, v_{j}\right)$ has to include some angular factor $A_{M_{X}}$, similar to $\mathrm{d} \Omega /(4 \pi)$ in Eq. (20), but somehow more complicated. Hence, we have

$$
\begin{aligned}
& I_{M_{X}}^{i}\left(\xi, v_{j}\right)=\frac{4 \pi}{A_{M_{X}}} \int \prod_{j=1}^{5} \mathrm{~d} \Omega_{j} \int_{0}^{\infty} \mathrm{d} x_{v} x_{v}^{2}\left[\prod_{j=1}^{5} \int_{-\infty}^{+\infty} \mathrm{d} x_{j} f_{j}\right] \\
& \times \delta\left(\sum_{j=1}^{5} \boldsymbol{p}_{j}\right)\left[\delta\left(x_{v}-\xi-\sum_{j=1}^{5} z_{j}\right)-\delta\left(x_{v}+\xi-\sum_{j=1}^{5} z_{j}\right)\right]
\end{aligned}
$$

where we have introduced a condensed notation for all particles, with exception of the neutrinos: $j$ is the index of the particles with $j=1 \ldots 5$, respectively, corresponding to $n, p, N_{i}, N_{f}, e$. With this notation, $\prod_{j=1}^{5} \mathrm{~d} \Omega_{j}$ is a global angular element of integration, while all other notations $\left(z_{j}, f_{j}\right)$ agree with previously defined notations with the convention that for a non-superfluid nucleon or an electron $z_{j} \equiv x_{j}$. Finally, notice that the $4 \pi$ factor in front of the expression comes from the integration over possible directions of the neutrino momentum.

To complete this section, we will give more advanced analytical formulae corresponding to some specific cases we will deal with in the following, not only because they are the easiest to treat numerically, but also because it is sufficient due to the still huge error-bars concerning dense matter microphysics. Those cases are

- the neutron branch with isotropic superfluidity of protons (gap A);

- the proton branch with any superfluidity of neutrons;

- the neutron branch with isotropic superfluidity of neutrons (gap A).

In the first case, due to full isotropy, one can write

$$
I_{M_{n}}^{p A}\left(\xi, v_{p}\right)=\frac{1}{g(\xi)} \int_{-\infty}^{+\infty} \mathrm{d} x_{p} f\left(z_{p}\right)\left(H\left(z_{p}+\xi\right)-H\left(z_{p}-\xi\right)\right)
$$

with

$$
\mathrm{H}(x)=\int_{0}^{+\infty} \mathrm{d} s s^{2} \frac{s-x}{\exp (s-x)-1}\left((s-x)^{2}+4 \pi^{2}\right)
$$

and

$$
g(\xi) \widehat{=} \xi \frac{367 \pi^{6}}{252}\left(1+\frac{189 \xi^{2}}{367 \pi^{2}}+\frac{21 \xi^{4}}{367 \pi^{4}}+\frac{3 \xi^{6}}{1835 \pi^{6}}\right) .
$$

In the second case, one has

$$
\begin{aligned}
I_{M_{p}}^{n X}\left(\xi, v_{n}\right)= & \frac{1}{g(\xi)} \int_{0}^{1} \mathrm{~d} c \int_{-\infty}^{+\infty} \mathrm{d} x_{n} f\left(z_{n}\right) \\
& \times\left(H\left(z_{n}+\xi\right)-H\left(z_{n}-\xi\right)\right),
\end{aligned}
$$

where $\mathfrak{c} \widehat{\equiv} \cos (\vartheta)$ appears in the $y_{n}$ variable while $g(\xi)$ and $H(x)$ are the same as in Eq. (26).

The third case leads to an integral to evaluate numerically that is more complicated, since analytical integration can be performed only on the electron and non-superfluid nucleon variables. With the usual technique, one gets

$$
\begin{aligned}
I_{M_{n}}^{n A}\left(\xi, v_{n}\right) & =\frac{1}{I_{M}(\xi)} \int_{-\infty}^{+\infty} \mathrm{d} x_{n 1} \mathrm{~d} x_{n 2} \mathrm{~d} x_{n 3} f\left(z_{n 1}\right) f\left(z_{n 2}\right) f\left(z_{n 3}\right) \\
& \times\left(\mathcal{H}\left(z_{n 1}+z_{n 2}+z_{n 3}+\xi\right)-\mathcal{H}\left(z_{n 1}+z_{n 2}+z_{n 3}-\xi\right)\right),
\end{aligned}
$$

with

$$
\mathcal{H}(x) \widehat{=} \int_{-\infty}^{+\infty} \mathrm{d} s s^{2} \frac{s-x}{\exp (s-x)-1},
$$

and where the normalisation function $I_{M}(\xi)$ was defined in Eq. (13).

For the numerical calculation, as was done for Durca reactions (see Eq. (23)), all those integrals for Murca reactions are cut in pieces in such a way that the $x_{i}$ variables take only positive values. However, we shall not give here more details on the algorithms that we used, and we send the reader to the Appendix or to the subroutine comments on http://luth2 .obspm.fr/ etu/villain/Micro/ Reduction.html. Instead, the next section will now describe the main numerical results.

\section{Numerical results}

Due to the complexity of the multidimensional integrations, we decided to test several numerical integration algorithms (see the Appendix for more details) and finally we obtained results in perfect agreement in the physical parameter range of interest. Here, we shall just give a graphical overview of those results for

- Durca reaction with superfluidity of each type;

- Murca reaction with isotropic superfluidity of the nonspectator nucleon (e.g. proton for the neutron branch);

- Murca reaction with isotropic superfluidity of the spectator nucleon (e.g. neutron for the neutron branch).

The first graph (Fig. 1) depicts in a three-dimensional way the reduction factor for the simplest situation, which is Durca reaction with isotropic superfluidity (of protons). We shall not give 


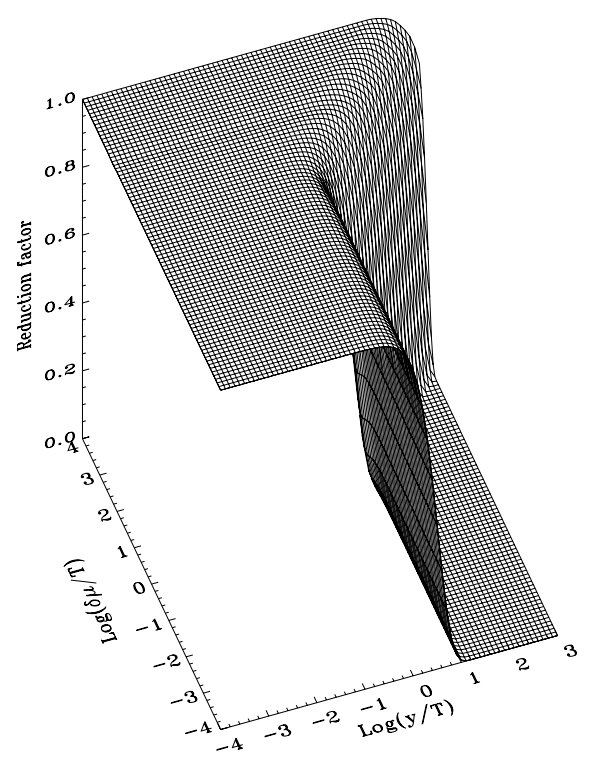

Fig. 1. Three dimensional view of the reduction factor for Durca reaction with isotropic superfluidity of the protons. Remember that here by $T$ we mean $k_{\mathrm{b}} T$ due to the chosen units.

Reduction Factor for several values of $\log _{10}(\mathrm{gap} / \mathrm{T})$ Durca Isotropic

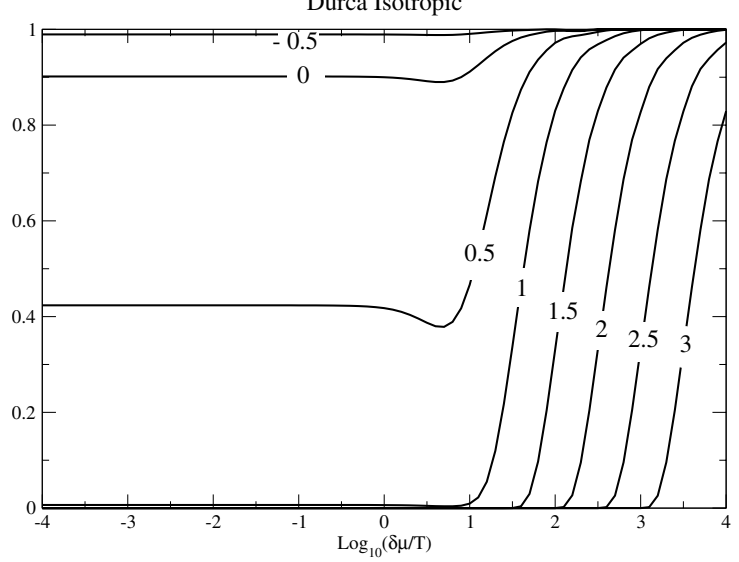

Fig. 2. Reduction factor as a function of the departure from beta equilibrium for several values of the gap's amplitude. Durca reaction with isotropic superfluidity of the protons. Remember that here by $T$ we mean $k_{\mathrm{b}} T$ due to the chosen units.

more 3D figures like this one, because they are not the best to look at the influence of the type of superfluidity, even if they help to have a quick idea of the effect of superfluidity. Indeed, this figure shows that for huge departures from equilibrium, superfluidity really has an impact only when the amplitude of the gap is of the order of $\delta \mu$, or is much larger than $\delta \mu$. In the first case, there is an exponential decay of the reduction factor, which leads, for the second case, to the expected "frozen composition" (reduction factor equal to 0 ). Yet, for very small values of the gap's amplitude (in respect to the temperature), the ratio with $\delta \mu$ does not matter and superfluidity is not relevant since the reduction factor is very close to 1 .

Figure 2 shows exactly the same results, but in another way. Here, the variable is $\delta \mu$, and each curve corresponds to a given value of the ratio gap/temperature. For huge gaps,

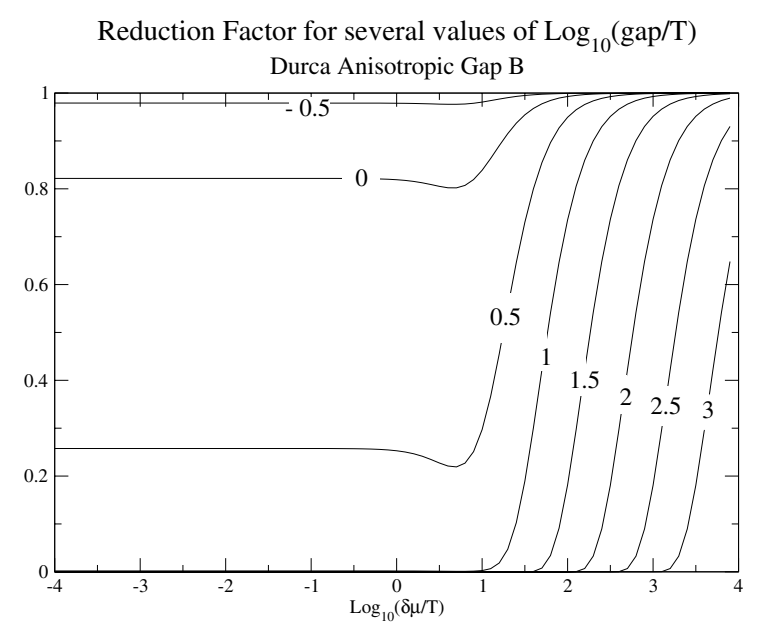

Fig. 3. Reduction factor as a function of the departure from beta equilibrium for several values of the gap's amplitude. Durca reaction with anisotropic superfluidity type B. Notation as in Fig. 2.

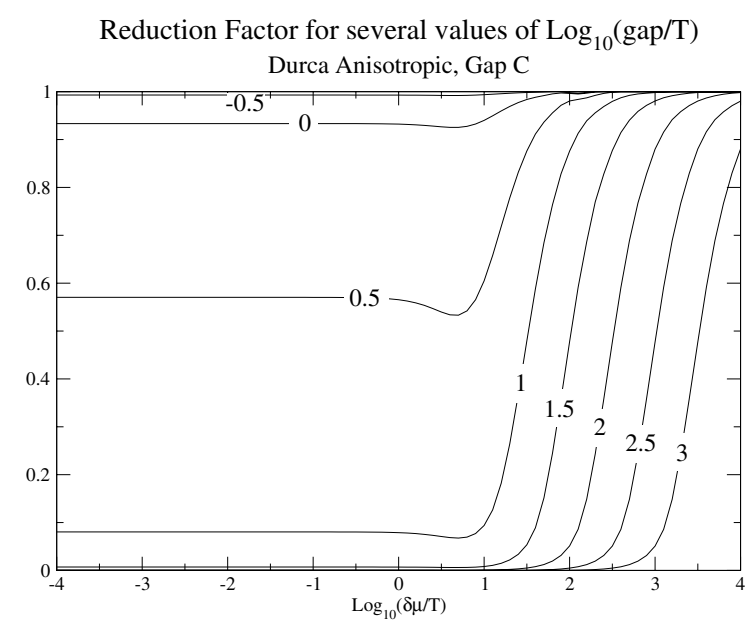

Fig. 4. Reduction factor as a function of the departure from beta equilibrium for several values of the gap's amplitude. Durca reaction with anisotropic superfluidity type C. Notation as in Fig. 2.

$\log _{10}(\mathrm{gap} / T) \geq 1$, it can be noticed that as soon as $\delta \mu \geq$ gap, and until the reduction factor becomes close to 1 , the curves are parallel. The meaning of this feature of the graphs is that, at least for that part of the curves, some functions $\mathfrak{f}$ and $\mathfrak{g}$ exist, such that the reduction factor can be written as $R \sim$ $f(g a p) g(g a p / \delta \mu)$. Furthermore, comparison of Figs. 3 and 4 with Fig. 2 enables to see that the effect of anisotropy of the gap cannot easily be evaluated since it can either increase (type C) or decrease (type B) the reduction factor in respect to its value in the isotropic case. However, what seems to be the most important is the maximal value of the gap on the Fermi surface since the value for case B is smaller than for case A, while for case $\mathrm{C}$ it is larger. As far as Murca reactions are concerned (Figs. 5 and 6), the situation is the following. If only one nucleon (the non-spectator one) is superfluid, then the difference with the Durca case seems very small and almost not visible (see Figs. 2 and 5). However, if the spectator nucleons (the most represented) are also superfluid the impact is much larger, as is depicted by Fig. 6. Nevertheless, it should not be forgotten that 


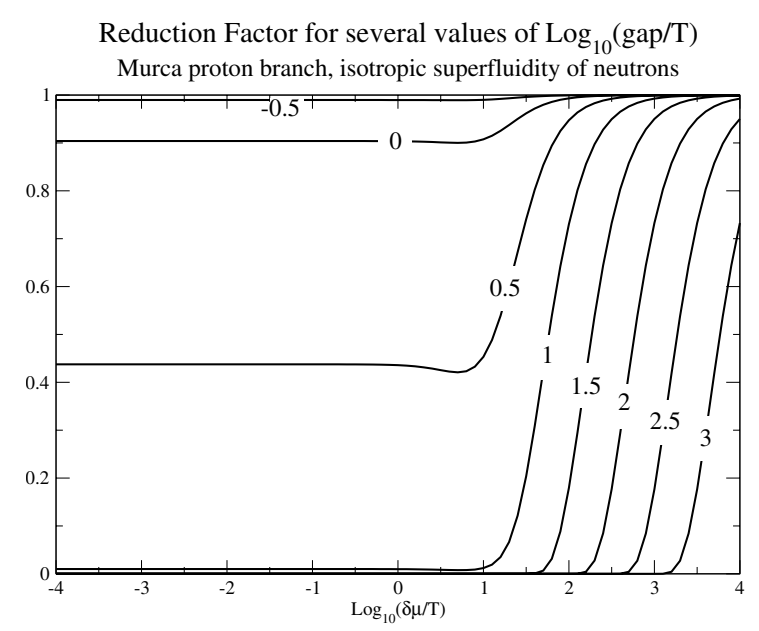

Fig. 5. Reduction factor as a function of the departure from beta equilibrium for several values of the gap's amplitude. Murca reaction with isotropic superfluidity of one single nucleon. Notation as in Fig. 2.

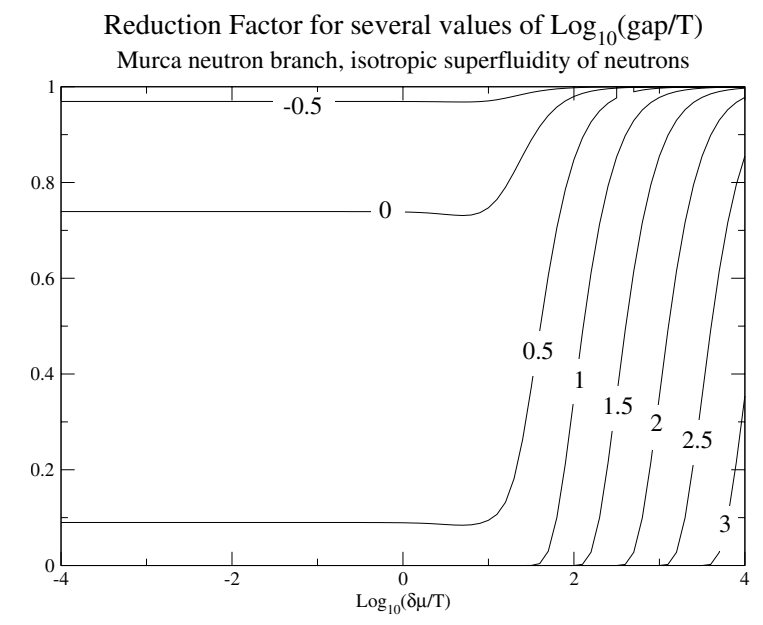

Fig. 6. Reduction factor as a function of the departure from beta equilibrium for several values of the gap's amplitude. Murca reaction with isotropic superfluidity of three nucleons out of four which are involved. Notation as in Fig. 2.

the reduction factors are calculated for relaxation times that are really different : Durca reactions allow for a much faster relaxation towards equilibrium (see, e.g., Sect. 3.5 of Yakovlev et al. 2001 and references therein).

\section{Discussion and conclusions}

We calculated the effect of nucleon superfluidity on the rates of beta processes in neutron star matter which is off beta equilibrium. Superfluidity reduces these rates by a factor that depends on the types of the beta process (direct Urca or modified Urca) and of superfluidity, but also on the dimensionless gaps and chemical-potential mismatch parameters $\Delta / k_{\mathrm{B}} T$ and $\delta \mu / k_{\mathrm{B}} T$, respectively. Due to the degeneracy of nuclear matter, the reduction factors do not depend explicitly on the EOS (see Sect. 4.1).

In order to reduce the number of parameters and the technical difficulties in calculating the integrals to evaluate, we assumed that we could always consider only one type of nucleon being superfluid, the larger gap prevailing. Such an approximation, which seems to be quite reasonable for the nuclear matter of NS cores (see Lombardo \& Schulze 2001), indeed allows to analytically integrate more variables (see Sect. 4), strongly reducing the computational time needed.

In the case of the direct Urca process, reduction factors values were calculated for the isotropic spin-singlet ${ }^{1} \mathrm{~S}_{0}$ pairing of protons and for two types of the angle-dependent gaps resulting from the spin-triplet ${ }^{3} \mathrm{P}_{2}$ pairing of neutrons. Since, in the modified Urca case, the phase-space integrals, which can be of up to the $12^{\text {th }}$ order, are very complex, we decided to first neglect the superfluid gaps anisotropy. This approximation can seems quite rough, however, it is supported by the fact that anisotropy of the gap can either increase or decrease the effect of superfluidity in respect to the case of the isotropic gap (see Sect. 5), while the actual state of the triplet neutron Cooper pairs is still poorly known (see Sect. 3).

Subroutines for the numerical calculation of the superfluid reduction factors are briefly described in the Appendix with link to the website on which they can be found. They yield the reduction factors for given $\Delta / k_{\mathrm{B}} T$ and $\delta \mu / k_{\mathrm{B}} T$. One has then to multiply by these factors the analytical expressions for the non-superfluid beta reaction rates to get the physical rates for superfluid matter.

Our results, combined with an equation of state of the neutron star core, can be used in numerical simulations of neutron star pulsations, to account for the dissipation due to the nonequilibrium beta processes implied by the local baryon density variations. Such a modeling will be valid both in a highly nonlinear suprathermal regime of dissipation $\delta \mu>k_{\mathrm{B}} T$ as well as in the $\delta \mu \ll k_{\mathrm{B}} T$ limit where the bulk viscosity description can be used (see Haensel et al. 2000, 2001, 2002). Another application concerns the calculation of non-equilibrium heating and neutrino emission of superfluid matter in a spinning down pulsar, which would allow one to model in a realistic manner the cooling/slowing-down of superfluid pulsars. Both problems will be studied by us in the near future.

The formulae presented in the present article were obtained under the simplifying assumption that neutrons, protons, and electrons move together as one single fluid, i.e., they are all at rest in one single reference system (local rest-frame). On a dynamic timescale, such an approximation is correct if all three fluids are normal (see, e.g., Villain et al. 2005). However, in the most general case of superfluid neutron and protons, we have to deal with three fluids, and therefore with three different flows of matter. First, we have a normal fluid composed of electrons and of normal components of neutrons and protons (this is a fluid of excited neutron and proton quasi-particles). Then, we have two flows of superfluid condensates, the neutron and proton one, connected between themselves by the superfluid entrainment (Andreev-Bashkin effect). If we connect the local rest-frame with normal fluid, then the equilibrium distributions of neutron and proton quasiparticles in the condensates should be calculated taking into account the relative velocities $\boldsymbol{u}_{n e}=\boldsymbol{u}_{n}-\boldsymbol{u}_{e}$ and $\boldsymbol{u}_{p e}=\boldsymbol{u}_{p}-\boldsymbol{u}_{e}$, respectively. For example, for isotropic gaps, in the rest-frame comoving with normal fluid, the neutron superfluid quasiparticle energies are $\varepsilon_{n}^{\prime}(\boldsymbol{p})=\varepsilon_{n}\left(\boldsymbol{p}-m \boldsymbol{u}_{n e}\right)+\gamma_{n n} \boldsymbol{p} \cdot \boldsymbol{u}_{n e}+\gamma_{n p} \boldsymbol{p} \cdot \boldsymbol{u}_{p e}$, where $m$ is 
neutron mass, and terms quadratic in $\boldsymbol{u}_{n e}$ and $\boldsymbol{u}_{p e}$ were neglected; the last two terms describe the entrainment effects and $\gamma_{\alpha \beta}(\alpha, \beta=n, p)$ is the microscopic entrainment matrix calculated by Gusakov \& Haensel (2005). Generally, macroscopic flows velocities are small compared to the Fermi velocities and lowest-order approximations are valid. The calculations in the three-fluid model will be the next step of our study of nonequilibrium beta processes in superfluid neutron star cores.

Acknowledgements. We are grateful to K.P. Levenfish for precious comments, and to J.-M. Chesneaux, F. Jézéquel and F. Rico for stimulating discussions. This work was partially supported by the Polish KBN grant No. 1-P03D-008-27 and by the Associated European Laboratory LEA Astro-PF of PAN/CNRS. LV also generously benefited from the hospitality of the Southampton University General Relativity Group during part of the development of this work.

\section{Appendix: Numerical technics}

The integrals presented in this article, which were to be evaluated numerically, are of quite standard type in the field of degenerate Fermi fluids. However, as they involve various mathematical features that can easily lead to numerical difficulties, we have estimated that it was worth interacting with some applied mathematicians expert in sophisticated algorithms to dynamically control the accuracy of numerical multi-dimensional integrals. These people, J.-M. Chesneaux, F. Jézéquel, F. Rico and M. Charrikhi from LIP6 of Paris 6 University, are part of the "Cadna team", Cadna being the acronym for "Control of Accuracy and Debugging for Numerical Applications" and also the name of the numerical tool they created (http://www-anp.lip6.fr/cadna/). We shall not give here a description of their algorithm, but let us just mention that it is based on discrete stochastic arithmetic, and allows for calculations with a chosen precision (see, e.g., Chesneaux \& Jézéquel 1998 and Jézéquel 2004).

Yet, the drawback of this method is that the time needed to carry out the computation can be very long, which prohibits to directly use it in physical simulations. As a consequence, we will no longer mention the Cadna code in the following, but it shall be noticed that we used it to test and calibrate our less sophisticated algorithms, and then to get clear ideas on the number of numerical points needed for various values of the parameters (see Jézéquel et al. 2005 and Charrikhi et al. 2002). Indeed, as we shall see now, the features of the integrals make that the needed number of points is not uniform in the $(v=$ $\left.\Delta / k_{\mathrm{B}} T, \xi=\delta \mu / k_{\mathrm{B}} T\right)$ plane.

The main difficulties in the integrations to perform are

- infinite size of the domains of integration;

- singularities in the denominators of some factors (e.g. $\mathrm{e}^{x}-1$ for $x=0$ );

- external free parameters (dimensionless gap amplitude $v$ and dimensionless departure from equilibrium $\xi$ ) that can be very large or different.

For not too precise calculations, the first of these issues is in fact quite easily dealt with, as using the asymptotic behaviours of exponential functions, one can show that it is not worth doing the integration on the full domain. Hence, the numerical domains are cut to some maximal values, which were chosen in such a way that exponential functions make further integration irrelevant. More precisely, due to the Fermi-Dirac distributions and to the "G-function" defined in Eq. (22), one can see that the typical relevant scale for the " $x$-variable" of a superfluid nucleon is $x \sim s_{x} \equiv \xi+\sqrt{2 v}$ and that the scale for the neutrino variable is $x_{v} \sim s_{v} \equiv \xi+v$. The maximal values for the numerical integration were 10 times the sum of 10 and of those typical scales, to prevent the occurence of problems for vanishing $v$ and $\xi$.

As far as singularities were concerned, we just dealt with them in putting in the code not exactly the functions but, beyond threshold values, some asymptotic formulae that are very good due to the exponential behaviours. The only trouble in this is that the presence of the possibly very large values of the $v$ and $\xi$ parameters can make the cut-off values very huge. However, the total number of numerical points needed was kept quite reasonable using

- some "logarithmic variables" (a suggestion done by V. Bezchastnov to K. Levenfish who kindly shared it with us): instead of directly integrating over the $x$-variables, we made the integrations over some $t$-variables defined as

$x \equiv s_{x}\left(\mathrm{e}^{t}-1\right)$,

where $s_{x}$ is the typical scale defined above;

- spectral decomposition: we evaluated the integrals with the so-called Gauss-Legendre quadrature method (see, e.g., Krylov 1962).

In this way, the main reason for needing huge numbers of numerical points were either an anisotropic gap with a very large amplitude, or small gap amplitude but combined with large departure from beta equilibrium. Anyhow, the subroutines used to produce the results displayed in Sect. 5 are gathered on the website http://luth2.obspm.fr/ etu/villain/Micro/ Reduction.html, together with some comments and tests, and they include the determination of reasonable numbers of numerical points for a wide range of the $v$ and $\xi$ parameters. Typically the proposed values (that can easily be changed playing in the subroutines) allow for a relative precision always at least better than $10^{-3}$ for $v$ in $\left[0,10^{3}\right]$ and $\xi$ in $\left[0,10^{4}\right]$. Of course, the subroutines can reach a much better precision just with a few additional numerical points and without huge change in the CPU time, but since there was no need for it, we limited the precision at that level.

\section{References}

Amundsen, L., \& Østgaard, E. 1985, Nucl. Phys., A442, 163

Andersson, A., \& Comer, G. L. 2001, MNRAS, 328, 1129

Charrikhi, M., Chesneaux, J.-M., Jézéquel, F., Rico, F., \& Villain, L. 2002, Proc. of the SCAN2002 conference, Paris (France), 23-27 September 2002

Chesneaux, J.-M., \& Jézéquel, F. 1998, J. Univ. Comp. Sci., 4, 2

Fernández, R., \& Reisenegger, A. 2005, ApJ, 625, 291

Friman, B. L., \& Maxwell, O. V. 1979, ApJ, 232, 541 
Gourgoulhon, E., \& Haensel, P. 1993, A\&A, 271, 187

Gusakov, M. E., \& Haensel, P. 2005, Nucl. Phys. A, 761, 333

Haensel, P. 1992, A\&A, 262, 131

Haensel, P., Levenfish, K. P., \& Yakovlev D. G. 2000, A\&A, 357, 1157

Haensel, P., Levenfish, K. P., \& Yakovlev D. G. 2001, A\&A, 372, 130

Haensel, P., Levenfish, K. P., \& Yakovlev D. G. 2002, A\&A, 381, 1080

Jézéquel, F. 2004, App. Num. Maths., 50, 147

Jézéquel, F., Rico, F., Chesneaux, J.-M., \& Charrikhi, M. 2005, Math. Comp. Simulation, to appear.

Krylov, V. I. 1962, Approximate calculation of integrals, ACM Monograph Series (New York, NY: The MacMillan Company)

Lattimer, J. M., Pethick, C. J., Prakash, M., \& Haensel, P. 1991, Phys. Rev. Lett., 66, 2701

Lee, U. 1995, A\&A, 303, 515

Levenfish, K. P., \& Yakovlev, D. G. 1994, Astron. Lett., 20, 43

Lindblom, L., \& Mendel, G. 1995, ApJ, 444, 804

Lindblom, L., Owen, B. J., \& Morsink, S. 1998, Phys. Rev. Lett., 80, 4843
Lombardo, U., \& Schulze, H.-J. 2001, in Physics of Neutron Star Interiors, ed. D. Blaschke, N. K. Glendenning, A. Sedrakian (Heidelberg: Springer), 30

Prix, R., \& Rieutord, M. 2002, A\&A, 393, 949

Reisenegger, A., \& Goldreich, P. 1992, ApJ, 395, 240

Reisenegger, A. 1995, ApJ, 442, 749

Reisenegger, A. 1997, ApJ, 485, 313

Shapiro, S. L., \& Teukolsky, S. A. 1983, Black Holes, White Dwarfs and Neutron Stars (New-York: Wiley-Interscience)

Urpin, V. A., \& Shalybkov, D. A. 1996, MNRAS, 281, 145

Villain, L., Bonazzola, S., \& Haensel, P. 2005, Phys. Rev. D, 71, 083001

Yakovlev, D. G., \& Levenfish, K. P. 1995, A\&A, 297, 717

Yakovlev, D. G., Levenfish, K. P., \& Shibanov, Yu. A. 1999, PhysicsUspekhi, 169, 825

Yakovlev, D. G., Kaminker, A. D., Gnedin, O. Y., \& Haensel, P. 2001, Phys. Rep., 354, 1 